\title{
Scintillating Fiber Detector for the Beam Loss Proton Measurements at J-PARC Linac
}

\section{Olga A. Konstantinova ${ }^{1}$}

High Energy Accelerator Research Organization

1-1 Oho, Tsukuba, Ibaraki, 305-0801, Japan

E-mail: olgaepost.kek.jp

\section{Hiroyuki Sako}

Japan Atomic Energy Agency

2-4 Shirakata-Shirane, Tokai, Naka-gun, Ibaraki 319-1195, Japan

E-mail: hiroyuki.sakoej-parc.jp

\section{Tomofumi Maruta}

High Energy Accelerator Research Organization

1-1 Oho, Tsukuba, Ibaraki, 305-0801, Japan

E-mail: tmaruta@post.j-parc.jp

\section{Akihiko Miura}

Japan Atomic Energy Agency

2-4 Shirakata-Shirane, Tokai, Naka-gun, Ibaraki 319-1195, Japan

E-mail: miura.akihikoljaea.go.jp

In the J-PARC linac, due to its high intensity $\mathrm{H}^{-}$beam, significant beam loss has been observed at a downstream straight beam line section called ACS (Annular-Coupled Structure linac). The loss is mainly due to a proton which is produced by double electron stripping of the $\mathrm{H}^{-}$beam by residual gas inside the beam pipe, and the titanium beam pipe. We have developed a detector system consisting of eight planes of the scintillating fiber detector in order to measure proton tracks emitted from the beam pipe of the J-PARC linac. The system measures the position and the time-of-flight of a charged particle track in a small solid angle. We show angular and energy distributions of proton tracks measured in 2012-2013. We also show comparison of the results with simulation.

Technology and Instrumentation in Particle Physics 2014

2-6 June, 2014

Amsterdam, the Netherlands

1

Speaker 


\section{Introduction}

Due to the high intensity $\mathrm{H}^{-}$beam, significant beam loss has been observed at the ACS (Annular-Coupled Structure linac) section in the J-PARC linac [1-4]. Its schematic layout and designed operation parameters achieved in 2012 are shown at Fig. 1 [1]. The highest radiation level was measured to be a few $100 \mu \mathrm{Sv} / \mathrm{h}$ in 2012. The beam loss distribution measured with Ar- $\mathrm{CO}_{2}$ proportional counters in the $z$-position (along the beam line), and simulated distribution are shown at Fig. 2. The peaks measured at $z=30-110 \mathrm{~m}$ are due to $\mathrm{X}$ rays emitted from RF cavities. High beam loss signals are detected in the ACS and L3BT sections.

The beam loss signal amplitude is supposed to be dependent on the residual gas pressure (see Fig. 3). The data obtained by the proportional counters is plotted as a function of the pressure in the beam duct. Thus we suggest that the beam loss is caused by electron stripping of the $\mathrm{H}^{-}$beam by residual gas in the beam duct.

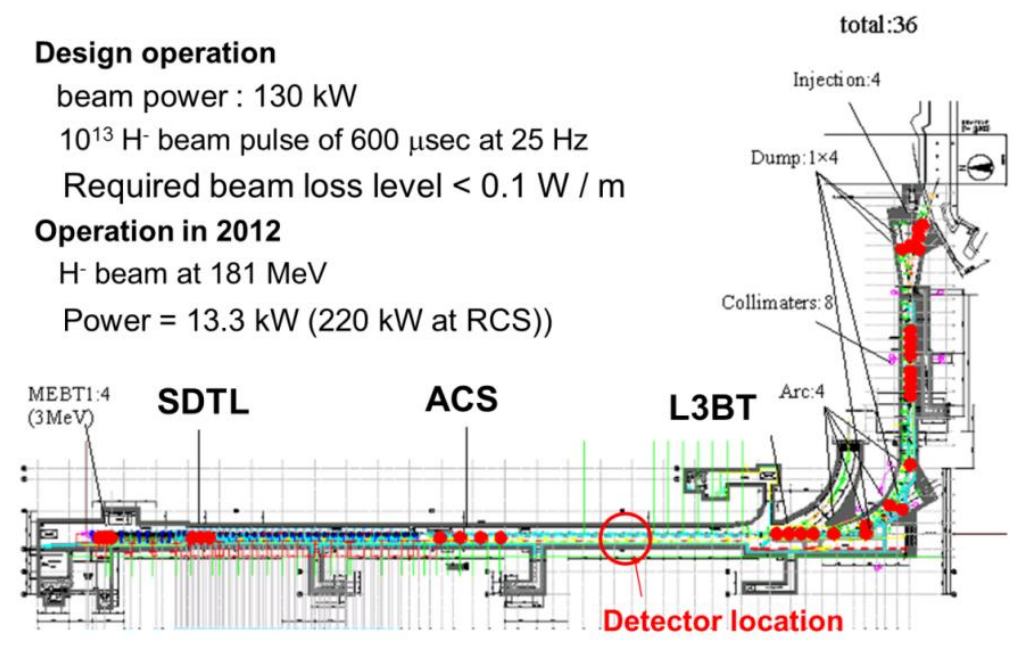

Figure 1: A schematic layout of the J-PARC linac.

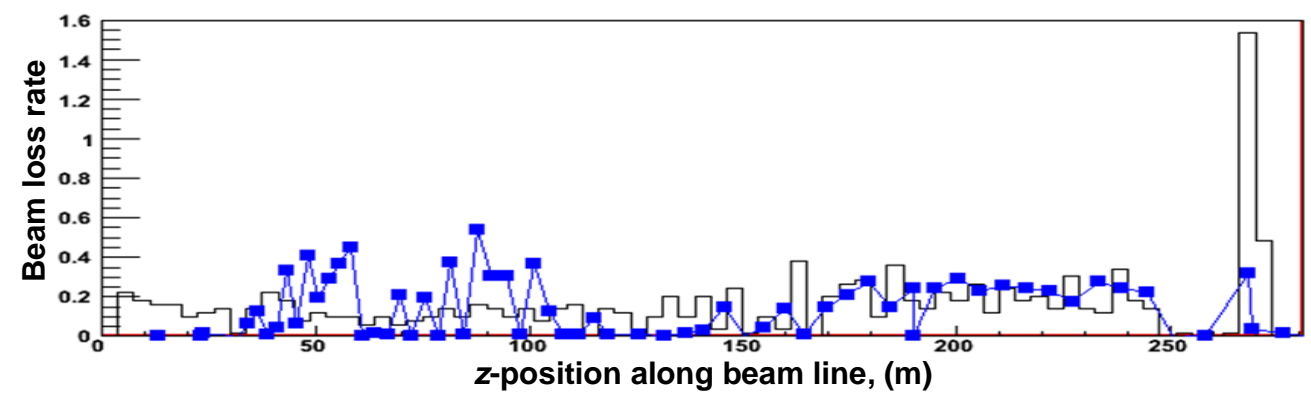

Figure 2: Beam loss measured with proportional counters (squares), and simulated beam loss (solid lines) as a function of the $z$-position along beam line $(\mathrm{m})$ at the J-PARC linac. 


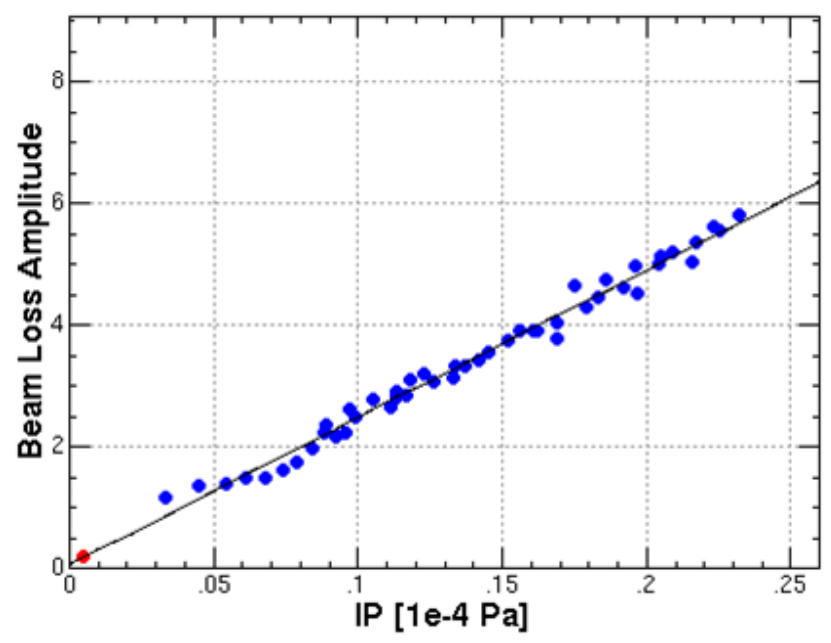

Figure 3: Beam loss signal amplitude measured with the proportional counter as a function of the pressure in the beam duct.

\section{Detector System}

The beam loss mechanism due to residual gas interaction of $\mathrm{H}^{-}$is shown ta Fig. 4 [5-6]. If an $\mathrm{H}^{-}$has interacted with an atom in residual gas, it is converted to $\mathrm{H}^{0}$ by losing an electron. Since $\mathrm{H}^{0}$ is a neutral particle, it is not focused by quadruple magnets and retains its momentum vector at the interaction since the binding energy between an electron and $\mathrm{H}^{0}$ is only a few eV.

Then, most of $\mathrm{H}^{0} \mathrm{~s}$ hit the beam duct. $\mathrm{An}^{0}$ passing through the beam duct loses one more electron with $100 \%$ probability to become $\mathrm{H}^{+}$. Thus, $\mathrm{H}^{+}$s are emitted from the surface of the beam duct.

As far as an $\mathrm{H}^{+}$is produced from an $\mathrm{H}^{-}$by double electron stripping, the measured number of $\mathrm{H}^{+} \mathrm{s}$ is same as the number of lost $\mathrm{H}^{-} \mathrm{s}$. The goal of the present detector system is to measure the number of $\mathrm{H}^{+}$s, namely protons emitted from the beam duct. The detector is required to measure a charged particle track in a high rate $\left(\sim 1 \mathrm{MHz} / \mathrm{cm}^{2}\right)$, and it has to distinguish a proton of 50-100 MeV from an electron of $\beta=1$. The detector system is designed as shown as Fig. 4 with eight detector planes made of plastic scintillating fibers. The upstream 4 planes are separated from the downstream 4 planes by about $1.6 \mathrm{~m}$ for the time-of-flight measurement. The upstream two planes measure horizontal positions and the other two planes measure vertical positions. Each plane consists of 16 of $4 \times 4 \times 16 \mathrm{~mm}^{3}$ scintillating fibers as shown in Fig. 5 . Each pair of planes is optically connected to a multi-anode photomultiplier (PMT) (Hamamatsu H8500C) via light guide fibers.

The detector system is installed in the upstream part of ACS section as shown in Fig. 6. The upstream and downstream sets of planes can be moved in the horizontal and vertical directions remotely with stepping motors in order to measure tracks at different positions and angles. PMT signals of each channel are transmitted via coaxial cables to the amplifiers at the $10 \mathrm{~m}$ above ground level. The trigger signal of a charged particle track is defined as a coincidence signal of the 12th dynode signals of the 4 PMTs. The dynode signal serves as 
analogue sum of signals in all the 64 channels of a PMT. Therefore, the trigger signal should be generated when a charged track passes through the 8 fiber planes. The trigger signal is used as a gate signal for the QDC (Charge-to-digital converter) to measure the signal amplitude, and used for the common stop signal of the TDC (Time-to-digital converter) to measure the signal timing.

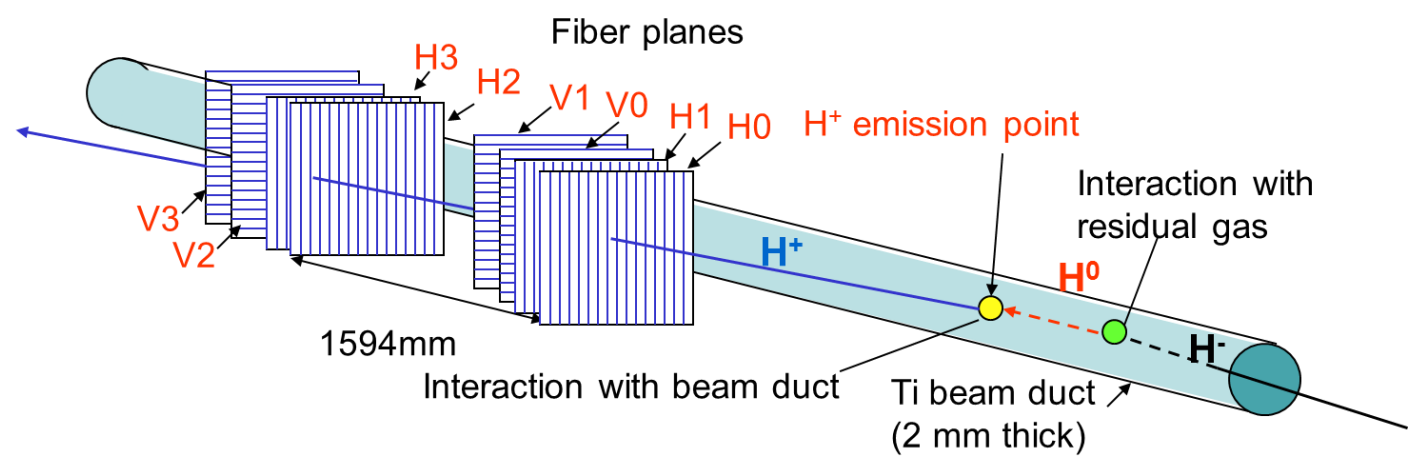

Figure 4: A schematic view of an $\mathrm{H}^{+}$detection with the detector system.

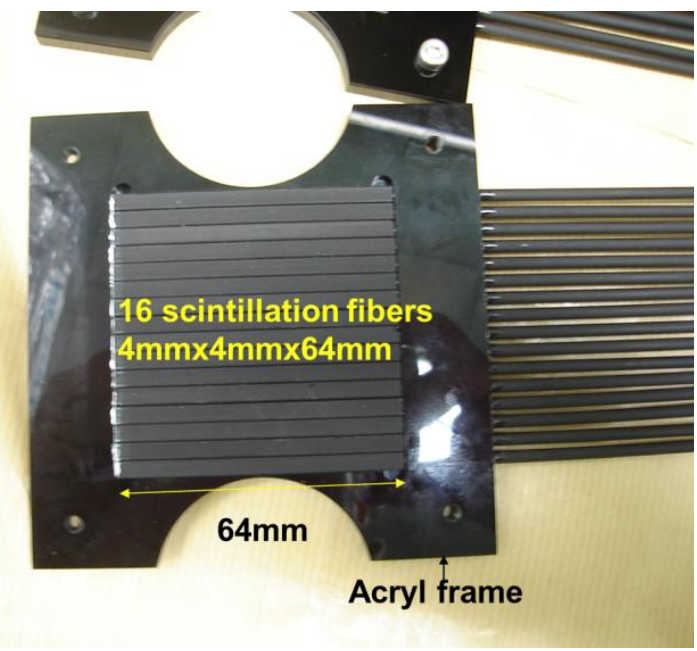

Figure 5: A scintillating fiber plane.

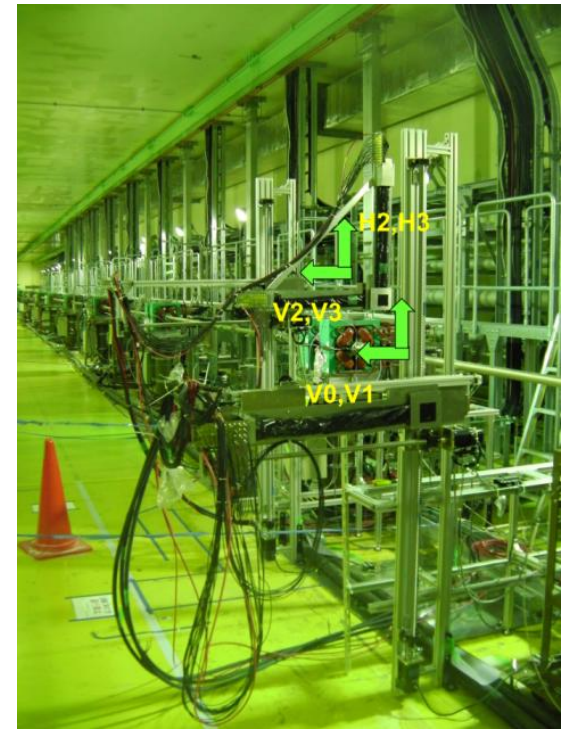

Figure 6: The detector system installed in the ACS section.

\section{Results}

We studied the possible background particles using the GEANT4 code to simulate the $\mathrm{H}^{-}$ electron stripping processes, which includes the geometry of the beam duct, magnets, and RF cavities, and the physics processes of $\mathrm{H}^{-}$and $\mathrm{H}^{0}$ electron stripping. Fig. 7 shows energy distributions of protons, neutrons, gamma-rays and electrons produced around the detector $z$ position. At energy above $60 \mathrm{MeV}$, proton signals are dominant, while at energy at 30-60 MeV, the main background is neutrons and the signal-to-background ratio is 1-5. Electrons and 
gamma rays have significant contributions only at energy less than $1 \mathrm{MeV}$. Since only a charged particle passing through the fiber planes can be detected, in the above background, only electrons can be background. In such case, the background can be rejected by requiring the time-of-flight cut for $\beta=0.31-0.43$ (corresponding to the energy of $50-100 \mathrm{MeV}$ ) for protons. A neutron could be detected if it has interacted with protons in the fiber and is converted to a proton which passes through the fiber planes. However, such a nuclear interaction probability is small $(\sim 2.8 \%)$.

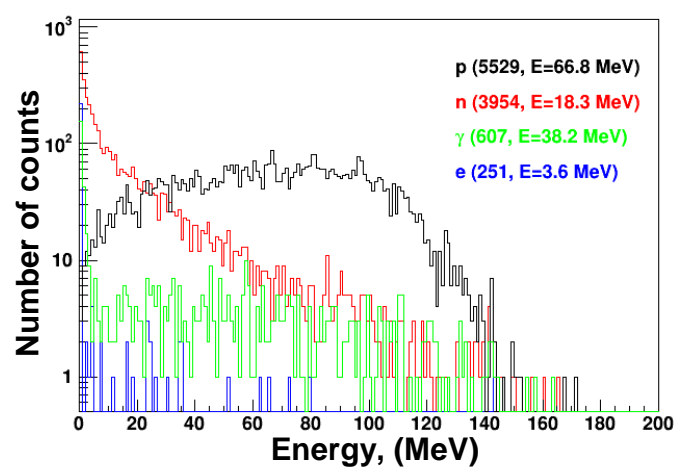

Figure 7: Simulated energy distribution for protons, neutrons, gammas, and electrons around the detector $z$-position.

Three-dimensional straight tracks were reconstructed in $(z, t, x)$ or $(z, t, y)$ space with horizontal 4 planes and vertical 3 planes respectively (signals at the vertical most downstream plane were not read out by electronics). We reconstruct a straight line connecting hits on the outmost planes, and search for hits on the inner planes closest to the track projection. Figure 8 shows the residual distribution at the $\mathrm{H} 2$ plane. Two signal peaks due to the fiber width are clearly seen.

Then, for each track, we calculate the time-of-flight as the time difference between a pair of planes as shown in Fig. 9. Only a single peak corresponding to protons is seen, while no peak corresponding to $\beta=1$ (to electrons) is observed. In order to extract the number of protons, we fit the distribution with a Gaussian function (accidental background) and with a Lorentzian function (signal) and obtained the number of protons as the integral of the fitted Lorentzian function.

The number of the protons per beam pulse (with $600 \mu$ s width and $25 \mathrm{~Hz}$ repetition) is derived using the following relation;

$$
N=\frac{N_{\text {track }}}{N_{\text {pulse }}} \frac{N_{\text {trig }}}{N_{D A Q}}
$$

where $N_{\text {track }}$ is the number of reconstructed proton tracks, $N_{\text {pulse }}$ is the number of beam pulses, $N_{\text {trig }}$ is the number of triggers per beam pulse, and $N_{D A Q}$ is the number of triggers collected by the data acquisition system.

The proton rate per beam pulse per solid angle is calculated as the following formula;

$$
\frac{d N\left(\theta_{x}\right)}{d \Omega}=\frac{d^{2} N}{\Delta \theta_{x} \Delta \theta_{y}}
$$


where $\theta_{x}\left(\Delta \theta_{x}\right)$ and $\theta_{y}\left(\Delta \theta_{y}\right)$ are the mean (rms) of the track angle distribution in the $z-x$ plane and the $z-y$ plane, respectively.

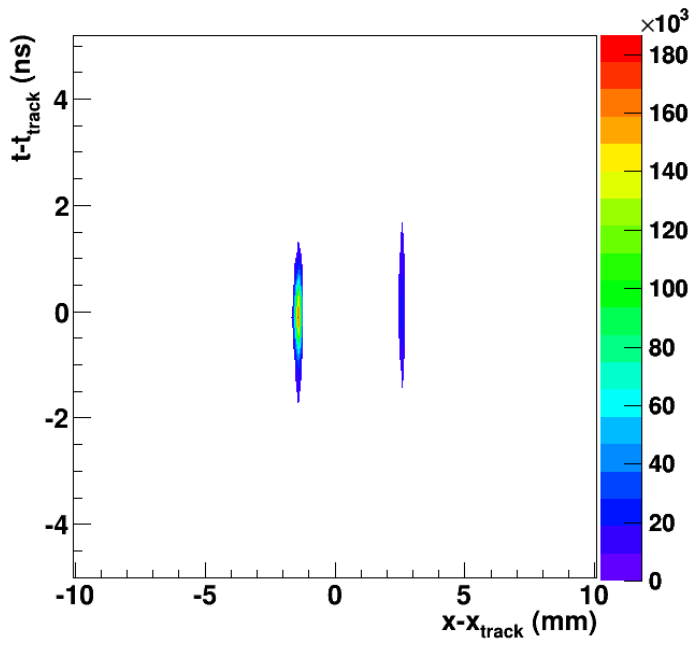

Figure 8: Residual distributions of a fiber hit in the horizontal position (horizontal axis) and the time (vertical axis) with respect to the projected track position at $\mathrm{H} 2$ plane.

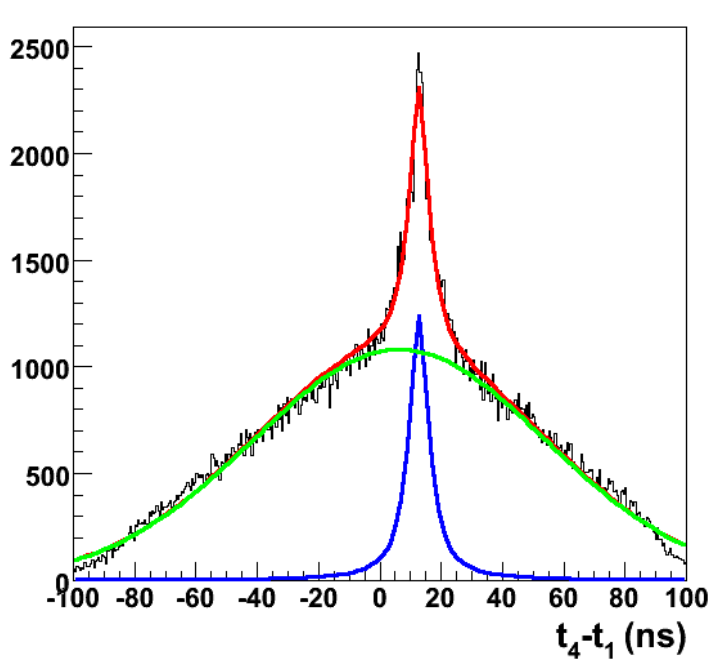

Figure 9: Time-of-flight distribution (ns) at $\theta_{x}=4.6$ deg. The signal peak (red line) corresponds to the protons, the blue line is its Gaussian fitting and the green line corresponds to the electrons signal.

Fig. 10 shows the proton rate $\left(\mathrm{deg}^{-2}\right)$ as a function of $\theta_{x}$. Each data point corresponds to the rate at a geometry configuration of the upstream and the downstream detector sets. Each color denotes $\theta_{x}$ dependence at a fixed $x$-position of the upstream detector set $\left(x_{u p}\right)$. The distributions peak around $\theta_{x}=5-6 \mathrm{deg}$. The simulated angle distributions shown as dashed lines also peak at 6 deg. correspondingly. The width of the measured distribution is consistent with the simulation at $x_{u p}=100 \mathrm{~mm}$, but smaller at $x_{u p}=300$ and $350 \mathrm{~mm}$, and wider at $200 \mathrm{~mm}$. Also the rates are not ordered in $x_{u p}$, but it rather decreases with the measured time (the order in time is $300 \mathrm{~mm}, 350$ $\mathrm{mm}, 100 \mathrm{~mm}$, and $200 \mathrm{~mm}$ ), which may suggest radiation damages of the scintillation fibers, but detailed investigation is necessary. Table 1 summarizes integrated rates over the solid angle per $z$-position $\left(\mathrm{m}^{-1}\right)$, and also the ratio of the measured number of $\mathrm{H}^{+} \mathrm{s}$ over that of $\mathrm{H}^{-} \mathrm{s}$ per beam pulse $\left(2.5 \times 10^{13}\right)$. The measured ratios are $27-67 \%$ of the estimated ratio from the residual gas pressure $\left(2.7 \times 10^{-8}\right)$. The smaller measured ratios may be due to inefficiency of the detector system which has not been corrected yet.

The mean proton energy as a function of $\theta_{x}$, shown at the Fig. 11 is compared with the simulation. The simulated energy of $\sim 90 \mathrm{MeV}$ is consistent with the measured energy within \pm $25 \mathrm{MeV}$ at $\theta_{x}=3-8 \mathrm{deg}$. A slight increase seen in the simulation at $\theta_{x}=2-5 \mathrm{deg}$. may be due to reduction of the beam loss because of smaller effective thickness of the titanium beam duct as $\theta_{x}$ increases. This tendency is seen at $x_{u p}=200,300$, and $350 \mathrm{~mm}$. Although at $\theta_{x}>8 \mathrm{deg}$. sudden decrease is predicted by the simulation, the data does not follow this and only slight decrease is observed, which has not been understood yet. 
The z-position of the closest approach of the track to the beam axis at an upstream position (see Fig. 12) should reflect the beam loss source position. At $x_{u p}=300$ and $350 \mathrm{~mm}$ the distributions peak around $z=4.5-4.0 \mathrm{~m}$ upstream from the detector system, while at $x_{u p}=100$ and $200 \mathrm{~mm}$ the distributions peak around $z=2.0-2.5 \mathrm{~m}$ upstream from the detector system. We need to apply efficiency and acceptance corrections to understand the source distributions.

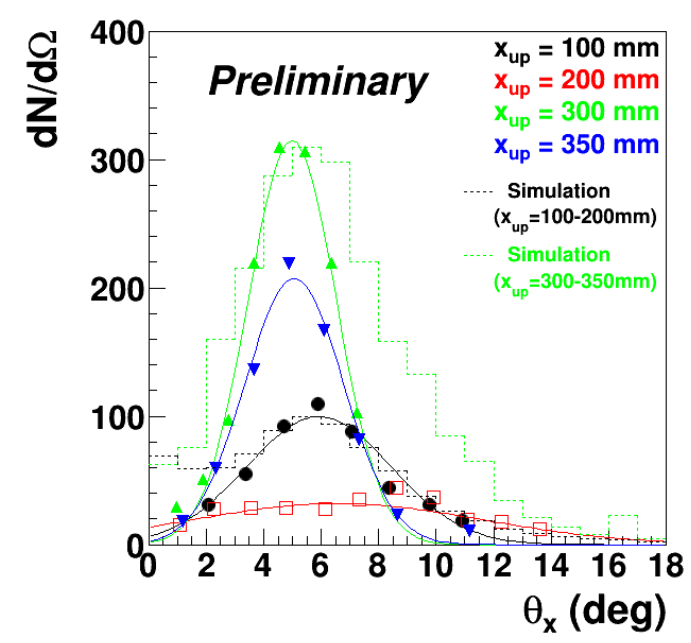

Figure 10: Proton rates per beam pulse as a function of $\theta_{x}$ (deg).

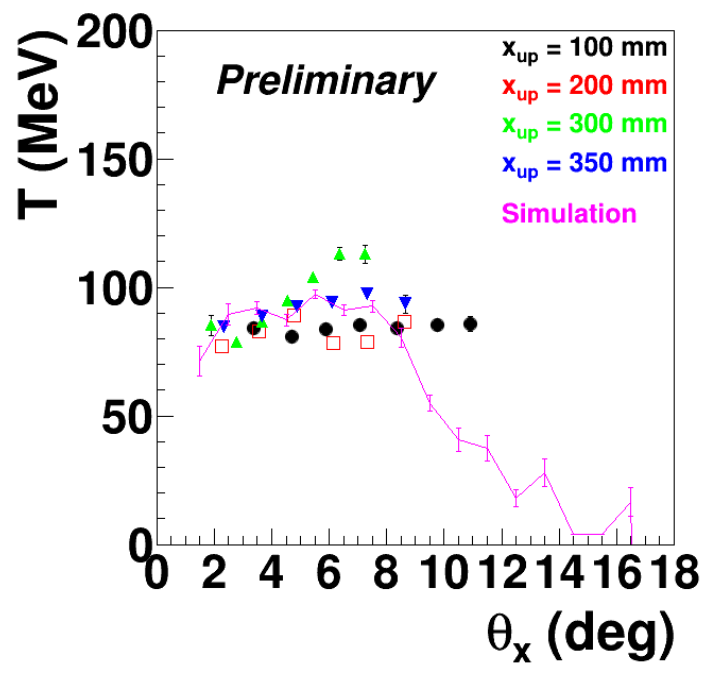

Figure 11: Mean kinetic energies $(\mathrm{MeV})$ as a function of $\theta_{x}$ (deg).

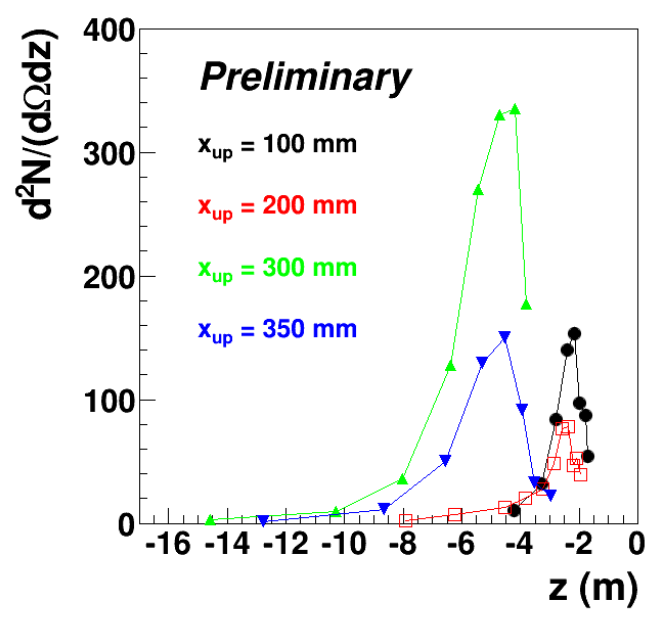

Figure 12: Proton rates as a function of the closest approach $z$-position $(\mathrm{m})$ of the track to the beam axis. 
Table 1: Proton rates per beam pulse and the $\mathrm{H}^{+} / \mathrm{H}^{-}$ratios.

\begin{tabular}{|l|l|l|}
\hline$x_{u p}(\mathrm{~mm})$ & $d N / d z\left(\mathrm{~m}^{-1}\right)$ & $N_{H+} / N_{H-}$ \\
\hline 100 & 316538 & $1.27 \times 10^{-8}$ \\
\hline 200 & 180189 & $7.21 \times 10^{-9}$ \\
\hline 300 & 441681 & $1.77 \times 10^{-8}$ \\
\hline 350 & 211315 & $8.45 \times 10^{-9}$ \\
\hline
\end{tabular}

\section{Summary and Prospects}

We measured the absolute beam loss rate for the first time at the J-PARC linac, by measuring $\mathrm{H}^{+}$emitted from the beam duct, using the scintillating fiber detector. We reconstructed charged particle tracks passing through seven fiber planes. The time-of-flight corresponds to the protons. We measured the proton rate per unit source length of (1.8-4.4) $\times 10^{5}$ $\left(\mathrm{m}^{-1}\right)$ and the corresponding $\mathrm{H}^{+} / \mathrm{H}^{-}$ratio of $(0.72-1.77) \times 10^{-8}$, which is close to the estimation from the residual gas pressure of $2.7 \times 10^{-8}$. The distributions of the horizontal angle $\theta x$ which have the peaks at 5-6 deg. are consistent with the simulation. The mean proton energies of 80 $115 \mathrm{MeV}$ are in a good agreement with the simulated value of $90 \mathrm{MeV}$ within $\pm 25 \mathrm{MeV}$.

We are going to study efficiency and acceptance of the detector and apply the corrections. We are also going to evaluate radiation effects. From Oct. 2014, we will restart proton measurements at downstream of the ACS section.

This work is supported by Grant-in-Aid for Exploratory Research No. 23656063 and Grant-in-Aid for Scientific Research (C) No. 24510134 from JSPS.

\section{References}

[1] H. Sako, et al., Beam Loss Particle Tracking in J-PARC Linac, in proceedings of PASJ2011 conference, 501 (2011).

[2] H. Sako, et al., Measurement of Beam Loss Tracks by Scintillating Fibers at J-PARC Linac, in proceedings of IPAC11 conference, 1251 (2011).

[3] H. Sako, et al., Observations of Beam Loss Proton Trajectories in J-PARC Linac, in proceedings of PASJ2012 conference, 638 (2012).

[4] H. Sako, et al., Beam Loss Track Measurements by a Fast Trigger Scheme at J-PARC Linac, in proceedings of LINAC2012 conference, 663 (2012).

[5] H. Sako, et al., Study of Beam Loss Spatial Distribution Measurements at J-PARC Linac, in proceedings of IPAC13 conference, 524 (2013).

[6] H. Sako, et al., Angular Distributions of Beam Loss Protons at J-PARC Linac, in proceedings of PASJ2013 conference, 1098 (2013).

[7] S. Agostinelli, et al., Geant4 - a simulation toolkit, Nucl. Instrum. Meth. A 506 (2003) 250 [10.1016/S0168-9002(03)01368].

[8] Flat panel type 64ch multianode PMT assembly, H8500C , Hamamatsu Photonics K. K., Literature Number: PMT:R10551-00-M64, www.hamamatsu.com. 